\title{
Phase Separation in Two-Dimensional Additive Mixtures
}

\author{
Arnaud Buhot and Werner Krauth * \\ CNRS-Laboratoire de Physique Statistique, Ecole Normale Supérieure, 24, rue Lhomond, \\ 75231 Paris Cedex 05, France
}

(Received June 12, 2021)

\begin{abstract}
We study 2-dimensional binary mixtures of parallel squares as well as of disks. A recent cluster algorithm allows us to establish an entropic demixing transition between a homogeneously packed fluid phase and a demixed phase of a practically close-packed aggregate of large squares surrounded by a fluid of small squares.
\end{abstract}

PACS numbers: 64.75.+g 61.20.Gy

Binary mixtures of impenetrable objects pose one of the important, and lively, problems of statistical physics. For many years it has been discussed whether objects of different types $l$ (large) and $s$ (small) would remain homogeneously mixed as the number of these objects per unit volume increases. Particularly interesting cases concern socalled additive mixtures [1], as hard spheres with radii $r_{l}$ and $r_{s}$ or cubes with length $d_{l}$ and $d_{s}$. The problem of phase separation in binary mixtures is of importance as the simplest model for colloids. It has been a meeting ground for many different theoretical, computational, and experimental approaches. As an example, the well-known closure approximations, as well as virial expansions, both of very great importance for the theory of simple liquids [2], have been brought to bear on this problem, often with contradictory results.

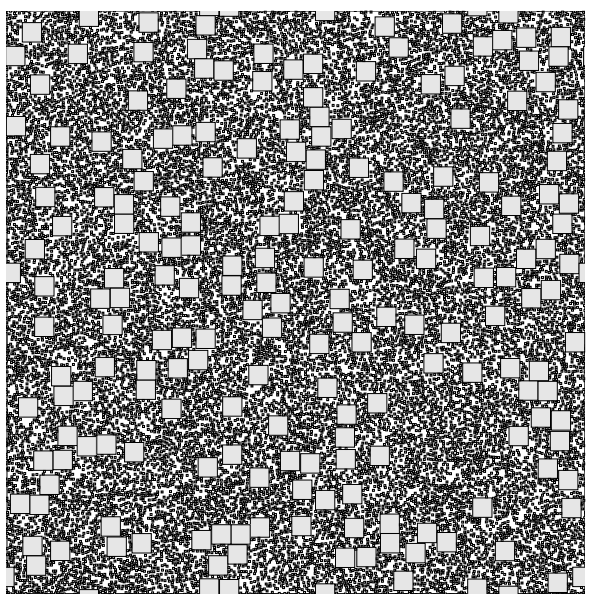

FIG. 1. Snapshot of 200 big squares $\left(d_{l}=1\right)$ and 20,000 small squares $\left(d_{s}=0.1\right)$ in a periodically continued box of size $30 \times 30$ (packing fractions $\eta_{l}=\eta_{s}=0.22$ ).

In this paper we discuss additive systems in two dimen- sions, parallel hard squares and also hard disks. For the parallel hard squares system, we clearly see a phase separation transition which has not been observed in previous work [3, 1 . F. For hard disks, we expect an analogous transition for extremely dissimilar sizes, which must satisfy $r_{s} / r_{l}<1 / 100$ for packing fractions $\eta_{l}=\eta_{s}<0.3$.

Monte Carlo simulations have long been performed on these systems. They were recently boosted by a new cluster algorithm [5.6], which allows thermalization of systems orders of magnitude larger than previously possible. The algorithm sidesteps a problem readily apparent in Fig. 1, which shows a typical configuration in the homogeneous phase. There, each large square is surrounded by very many small objects. Trial moves of large squares will therefore be rejected in the overwhelming majority of cases, and the algorithm will get stuck quickly. Our algorithm rather swaps large patches of the configuration in a way which preserves detailed balance 15 .7]. The algorithm is applicable for arbitrary shapes and in any dimension, and in continuous space as well as on the lattice. Most importantly, the method works even for objects very dissimilar in size as long as the total density is not too high.

We are able to converge our simulations of twodimensional parallel squares for total packing fractions $\eta=\eta_{s}+\eta_{l}$ which do not sensibly exceed the percolating density $\eta_{\text {perc }}$. As in $3 \mathrm{D}$, we notice that the density $\eta_{\text {perc }}$ depends very little on the ratio $R=d_{s} / d_{l} \leq 1$. We find $\eta_{\text {perc }} \simeq 0.5$. Figs 1 and 2 show snapshots of the simulations for 200 large squares and 20,000 small squares at equal composition $\left(\eta_{s}=\eta_{l}\right)$ and total packing fractions $\eta=0.44$ and $\eta=0.60$, respectively. It is evident that Fig. 1 represents a homogeneous mixture whereas the system shown in Fig. 2 consists of a 'solid' block of large squares surrounded by a fluid of small squares. In our opinion, these runs present direct evidence for a transition of the homogeneously mixed fluid into a (solid-fluid) phase.

*buhot@physique.ens.fr; krauth@physique.ens.fr 


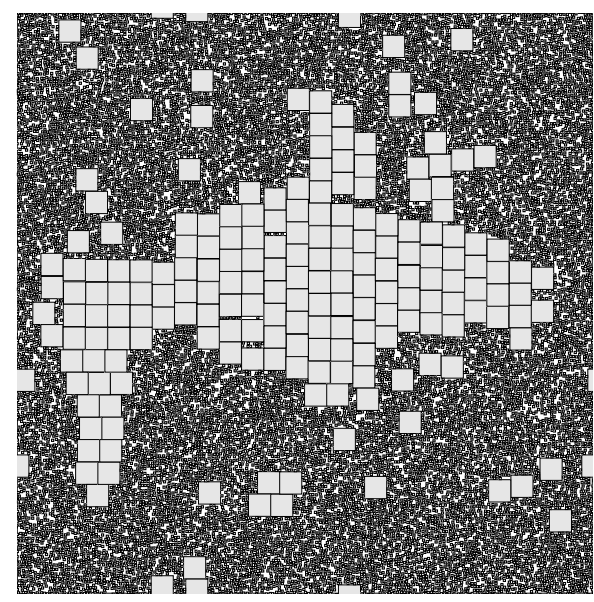

FIG. 2. Snapshot of 200 big squares $\left(d_{l}=1\right)$ and 20,000 small squares $\left(d_{2}=0.1\right)$ in a periodically continued box of size $26 \times 26$ (packing fractions $\eta_{l}=\eta_{s}=0.30$ ).

In the simulations at $\eta=0.6$, we have slightly exceeded the percolation threshold $\eta_{\text {perc }}$. Therefore, the algorithm will swap patches which usually comprise almost the whole system. This generates problems for large systems, and we have e.g. been unable to converge (at $\eta=0.6)$ a sample with $N_{l}=800, N_{s}=80,000$. In contrast, the simulations at lower packing fractions converge extremely rapidly for arbitrary system size. We can summarize the situation for equal composition $\left(\eta_{s}=\eta_{l}\right)$ by the diagram of Fig. 3: The gray area corresponds to the region of the diagram in which our algorithm performs extremely well. As mentioned, this region is delimited for the homogeneous system by the percolation threshold and by the appearance of high-density areas as a consequence of phase separation.

We also studied the instability line as a function of the composition $x=\eta_{l} /\left(\eta_{s}+\eta_{l}\right)$, especially since recent work of Cuesta [4] suggests symmetry of the phase diagram with respect to $x=0.5$ in the 3 -dimensional system. In the 2 -dimensional system, simulations at different compositions $(x=0.3,0.5$ and 0.7 for $R=0.1$ ) contradict this behavior since critical packing fractions are $\eta_{\text {crit }}=0.49 \pm 0.02,0.53 \pm 0.02$ and $0.60 \pm 0.05$, respectively.

In our three-dimensional simulation [6], it was impossible to interpret the data by direct inspection, as in Fig. 1 and Fig. 2. We analyzed the transition therefore with the help of the integrated pair correlation functions $G_{l l}(r)=4 \pi \eta_{l} \int r^{\prime} d r^{\prime} g_{l l}\left(r^{\prime}\right)$ [2]. We repeat the analysis in the $2 D$ case in order to stress the soundness of our procedure, which considers $G_{l l}$ rather than the much noisier $g_{l l} . G_{l l}(r)$ determines the average number of large particles around a given large particle within a distance $r$. For the special case of parallel hard squares, we define $r=\max (\Delta x, \Delta y)$, where $\Delta x$ and $\Delta y$ are the two (periodically continued) lateral distances. With this definition, the distance of two large squares in contact is $r=d_{l}$ and $G_{l l}=\eta_{l} \int_{\max (|x|,|y|)<r} g_{l l}(x, y) d x d y$ with $g_{l l}$ the usual pair correlation function [2]. In Fig. 4, one can see that the mixed system's $G_{l l}$ has pulled away from the pure system's correlation function on all scales, a clear indication that the large scale structure of the fluid has changed. The staircase structure is a very clear indication of solid order. Finally, the reason for the rather large finite-size effects at large separation is immediately clear, since $G_{l l}(r)$ has to meet the curve of the corresponding monodisperse system with $\eta=\eta_{l}$ for half the box length. A similar analysis was used to establish the instability line in Fig. 3.

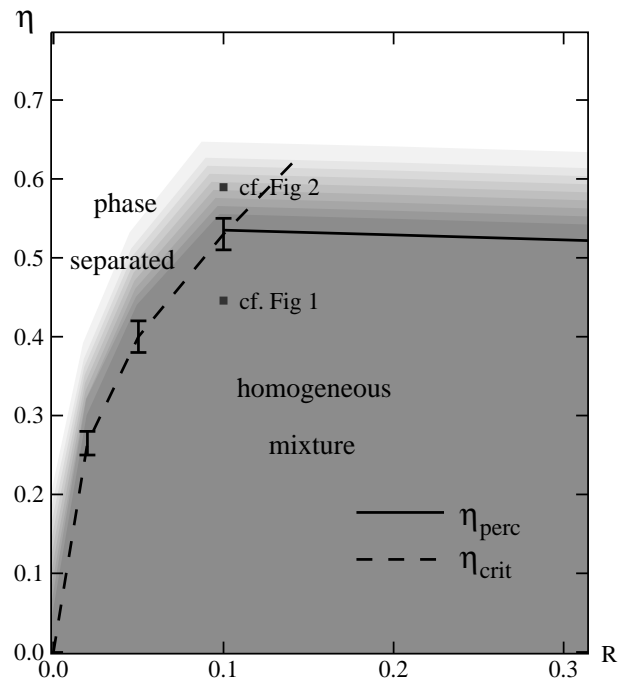

FIG. 3. Phases of the two-dimensional hard square system at equal composition $\eta_{s}=\eta_{l}$ (total packing fraction $\eta=\eta_{s}+\eta_{l}$ vs. $\left.R=d_{s} / d_{l}\right)$. The squares locate the parameters of the snapshots in Figs 1 and 2. The region in which our algorithm performs excellently is shaded in gray.

A comparison of Fig. 4 with the data for the homogeneous mixture (cf. Fig. 1) at $\eta=0.44$ is very reveiling. In the latter case (not shown), the difference between the mixed and the monodisperse system concerns mainly the region of small separation between the squares and would be unobservable on the scale of Fig. 4. The same observation was made in $3 \mathrm{D}$. The effects at small separations $r$ are already detectable by visual inspection of Fig. 1, since there are quite many 'bound' pairs and triplets. We find that each large square has bound an average of 0.8 squares. This agrees very nicely with our observation in $3 D$, where we noticed the onset of the phase-separation instability as the additional binding was close to one.

We also performed simulations for mixtures of hard disks. Let us begin our discussion with an indirect heuristic argument which connects transition parameters for squares and for disks. It has long been understood [1] that the overlap of excluded volumes entropically favors 
close contact of the large objects. The excluded volume (for a small square) around a big square consists in the area of the latter and a strip of width $d_{s} / 2$ around it. Side-to-side contact between two squares leads to an overlap of excluded volume of the size $\Delta V_{\text {square }} \sim d_{l} \times d_{s}$. As the large objects touch, the volume available to the small particles and therefore their entropy increase. At the same time, contact of large objects decreases their contribution to the entropy of the complete system. The phase separation transition appears when those two contributions are equals. As the decrease of entropy due to contact of large objects may be considered as independent of the ratio $R$ of the size of the small and large objects, we may compare the increase of entropy due to larger available volume for small disks or squares. In fact, since the small particles' contribution to the entropy is $S \sim N_{s} \log V_{s}$ (with $V_{s}$ the available volume), one finds $\Delta S \sim\left(N_{s} / V\right) \Delta V_{\text {square, }}$ and therefore

$$
\Delta S_{\text {square }} \sim \eta_{s} d_{l} / d_{s}
$$

Repeating the same calculation for disks, we notice of course that the overlap of excluded volume $\Delta V_{\text {disk }} \sim$ $\sqrt{r_{s}^{3} r_{l}}$ and the number of concerned small disks are much smaller. This leads to

$$
\Delta S_{\mathrm{disk}} \sim \eta_{s} \sqrt{r_{l} / r_{s}}
$$

This order-of-magnitude argument tells us that (with a hard square transition at $d_{s} / d_{l} \sim 1 / 10$ for $\eta \simeq 0.5$ ) we can expect a transition for disks at best for $r_{s} / r_{l} \sim 1 / 100$. Accordingly, our simulations for $r_{s} / r_{l} \gtrsim 1 / 100$ at $\eta=0.6$ have reveiled no instability of the homogeneous phase. Even larger simulations, at $r_{s} / r_{l}=1 / 150$, (50 large and 1, 250, 000 small disks, $\eta=0.6$ ) did not converge, even though the additional binding has continuously increased in the course of a month-long simulation. In these simulations, the depletion potential is very strong, but also extremely short-ranged. The Monte Carlo simulation of such 'golf-course' potentials is of course extremely timeconsuming and often impossible.

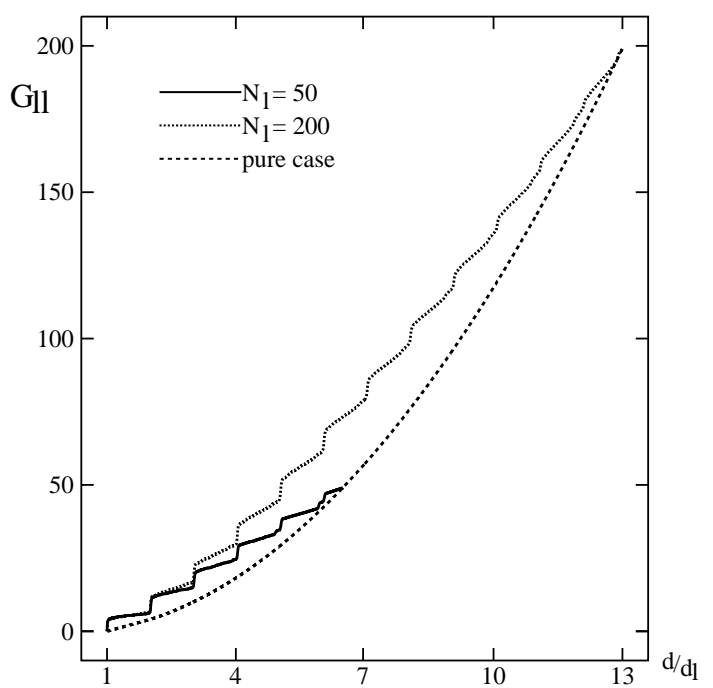

FIG. 4. Integrated two-point correlation function for the dense system $\eta_{l}=\eta_{s}=0.30$ (cf. Fig. 2) for 50 and 200 large squares, respectively. The two curves are compared to the monodisperse system's correlation function.

In conclusion we have studied the problem of phase separation of two-dimensional systems (hard squares, hard disks) by direct Monte Carlo simulation. For hard squares, our Monte Carlo data leave little room to doubt a direct fluid to (solid-fluid) transition. For hard disks, the stability of the homogeneous mixture seems established for any 'reasonable' ratio of radii $r_{s} / r_{l} \gtrsim 1 / 100$. Our heuristic argument would however lead us to expect a transition for even more extreme ratios.

[1] S. Asakura and F. Oosawa J. Chem. Phys. 221255 (1954).

[2] J. P. Hansen and I. R. Macdonald Theory of Simple Liquids, 2nd edition (Academic, London, 1986).

[3] M. Dijkstra, D. Frenkel and J. P. Hansen J. Chem. Phys. 101, 3179 (1994); M. Dijkstra and D. Frenkel Phys. Rev. Lett. 72298 (1994).

[4] J. A. Cuesta Phys. Rev. Lett. 763742 (1996).

[5] C. Dress and W. Krauth J. Phys. A: Math Gen 28, L597 (1995).

[6] A. Buhot and W. Krauth Phys. Rev. Lett. 803787 (1998).

[7] For a pedagogical discussion, cf. H. Gould, J. Tobochnik, L. Colonna-Romano Computers in Physics 11157 (1997). A successful application in a 3-dimensional lattice gas model was presented in J. R. Heringa and H. W. J. Blöte Physica 232A, 369 (1996). 